Original Research Paper

\title{
Improved Steering and Adaptive Cruise Control for Autonomous Vehicles Using Model Predictive Control
}

\author{
Sai Charan Dekkata and Sun Yi \\ Department of Mechanical Engineering, North Carolina A\&T State University, Greensboro, USA
}

Article history

Received: 19-02-2019

Revised: $12-03-2019$

Accepted: 11-06-2019

Corresponding Author:

Sai Charan Dekkata

Department of Mechanical

Engineering, North Carolina

A\&T State University,

Greensboro, USA

Email: sdekkata@aggies.ncat.edu

\begin{abstract}
The research on autonomous vehicles was started nearly a century ago but the major parts of significant advance have been done over the past few decades. In this paper, designing and simulating of a Model Predictive Controller (MPC) and comparing it to a classical feedback controller, which was designed and implemented on a car for Lane Keeping Assist (LKA) and Adaptive Cruise Control (ACC) systems, are presented. The goal of the control system is to follow linear trajectories and stay in the lanes by correcting the lateral deviation to reach the destination point. By regulating the longitudinal and lateral accelerations of the vehicle, it is possible to provide hands-free driving experience only on highways as it satisfies the level 2 autonomy according to the SAE automation levels. In the Adaptive Cruise Control (ACC) system, the ego car follows the velocity set by driver until it maintains a safe distance from the lead car. If the space between the lead car and the ego car is less than the safe distance, the ego car reduces the velocity and does not follow the driver set velocity until it has reached a safe distance with the lead car. The frontal or rear-end collisions and traffic congestions can be reduced by maintaining the safe distance using the spacing control by Adaptive Cruise Control (ACC) system. Model Predictive Control with the linear time invariant system with input, output and state variables uses the feed-forward and the disturbance models are used for ACC and Lane keeping Assist systems.
\end{abstract}

Keywords: Steering Control, Lane Keeping Assist, Adaptive Cruise Control, Model Predictive Control, Autonomous Vehicle

\section{Introduction}

The technologies have been growing rapidly with the increase in the driver safety features like Intelligent Driver's Assist Systems (IDAS) and Advanced Driver Assist Systems (ADAS) which try to help the driver to travel safely. Still due to lack of proper usage and the stress drivers undergo in long drives lead to numerous accidents in the past decades. In 2015 over 1.3 million deaths occurred on the roads in the entire world. According to the National Highway Traffic Safety Administration (NHTSA) under the Fatality Analysis Reporting System (FARS) the number of deaths occurred in United States of America is 37,461 in 2016. For making driving safer with the development of active safety systems like Anti-lock Braking Systems (ABS), Electronic Stability Program (ESP), Vehicle Stability Program (VSP) and the Traction Control (TC). Even though all these systems improve the vehicle's behavior when an unexpected scenario arises, they do offer help to the driver which was very minimal which reduces the crashes only up to $10-15 \%$.

In the most recent developments, the active safety has become high priority and the safety standards have been improved a lot. The inclusion of sensors such as cameras, LiDAR, Radars tend to improve the safety of the vehicle by detecting the objects way further and taking necessary actions in avoiding the objects and staying within the designated lanes. But one of the more reliable development would be autonomous driving systems, even though they have pros and cons. Considering the ability of the system to drive autonomously controlling the steering, acceleration, braking, detecting and avoiding the objects would be a major development in the automotive industry.

This paper presents development of the Model Predictive Control (MPC) for steering of an autonomous vehicle. A Proportional Integral Derivative (PID) controller was designed and implemented for the 
autonomous vehicle for steering and ACC. The design and simulation were done using MPC and comparing the results with the PID controller. As MPC can be used for future predictions and considering the system operating constraints which makes it more suitable than the PID for the autonomous driving systems.

\section{Model Predictive Control}

This paper mainly concentrates on the Model Predictive Control (MPC) for steering and Adaptive Cruise Control systems for an autonomous vehicle. PID controller has been designed and implemented for the autonomous vehicle for steering control and Adaptive Cruise Control. The design and simulation were done using MPC and comparing the results with the PID controller. As MPC can be used for future predictions and considering the system operating constraints which makes it more suitable than the PID for the autonomous driving systems.

Figuer 1, the overview of the Model Predictive Control schematic has been presented. Models are required for prediction and thus logically are tackled first. To automate MPC, it is necessary to present the concepts in mathematical terms. Simple manipulation and algebra require linear models as the superposition can be used. Typical linear models are transfer function or state-space. To some extent step response models are subset of transfer functions. MPC encompasses a range of control methods used in single-input singleoutput and multiple-input and multiple-output processes. (Yiqi Gao, 2014).

Process model:

$$
x_{k+1}=A x_{k}+B u_{k}
$$

$$
y_{k}=C x_{k}+D u_{k}
$$

Complex dynamical systems behaviors are generally represented by the models used in MPC, it requires model to minimize the difference between predicted output and desired output. If there are no constraints in Linear MPC, it reduces to linear quadratic regulator. Minimize:

$$
\left\|y_{t}-y\right\|_{n}
$$

Constraints:

$$
\begin{aligned}
& y_{\min } \leq y \leq y_{\max } \\
& \Delta u_{\min } \leq y \leq \Delta u_{\max } \\
& u_{\min } \leq y \leq u_{\max }
\end{aligned}
$$

Optimisation Criterion:

$$
\begin{aligned}
& =\sum_{k=0}^{N_{p}}\left\{Q_{1}\left[e_{1}\left(t_{k}\right)\right]^{2}+\ldots .+Q_{n}\left[e_{n}\left(t_{k}\right)\right]^{2}\right\} \\
& +\sum_{k=1}^{N_{c}}\left\{R_{1}\left[\Delta u_{1}\left(t_{k}\right)\right]^{2}+\ldots .+R_{r}\left[\Delta u_{r}\left(t_{k}\right)\right]^{2}\right\}
\end{aligned}
$$

\section{Proportional Integral Derivative}

Proportional term considers how far the measured process variable from the set point at any instant in time and adds or subtracts from controller bias value. Integral term address how long and how far the measured process value has been from set point continually summing the controller error over time. Derivative term considers how fast the controller error is changing at any instant using the rate of change or slope of the error curve. Rapidly changing $e(t)=$ large derivative $=$ large impact on controller output signal. Derivative doesn't matter if the controller error is positive, negative or how much time has been passed, it considers only how fast the controller error is changing:

$$
u=u_{0}+K_{p} e+\frac{K_{i}}{T_{i}} \int_{0}^{t} e(t) d \tau+K_{d} T_{d} \frac{d e(t)}{d t}
$$

Figuer 2, the overview of the Proportional Integral Derivative schematic has been presented. A derivative is a slope or rate of change, where $u$ is controller output signal, $u_{0}$ is the controller bias value, $T_{d}$ provides a separate weight to the derivative (or rate $o$ change) of error, control error $=$ setpoint - measured process variable $(e(t)=S P-P V) . T_{d}$ has units of time so it is always positive and larger values of $T_{d}$ increase influence on the derivative term: (Dave Misir, 1994)

$u_{k}=K_{p} e_{k-1}+K_{d}\left(\frac{e_{k-1}-e_{k-2}}{T}\right)$

In $z$ domain, it can be equivalently written as:

$C=\frac{u}{e}=K_{p} \frac{1}{z}+K_{d}\left(\frac{z-1}{T z}\right)=\frac{\left(K_{p} T+K_{d}\right) z-K_{d}}{T z}$

$$
\begin{aligned}
& \frac{Y}{R}=\frac{\frac{z+3}{z^{2}-2 z} \frac{\left(K_{p} T+K_{d}\right) z-K_{d}}{T z}}{1-\frac{z+3}{z^{2}-2 z} \frac{\left(K_{p} T+K_{d}\right) z-K_{d}}{T z}} \\
& \Rightarrow \frac{(z+3)\left(\left(K_{p} T+K_{d}\right) z-K_{d}\right)}{T z\left(z^{2}-2 z\right)-(z+3)\left(\left(K_{d} T+K_{d}\right) z-K_{d}\right)}
\end{aligned}
$$




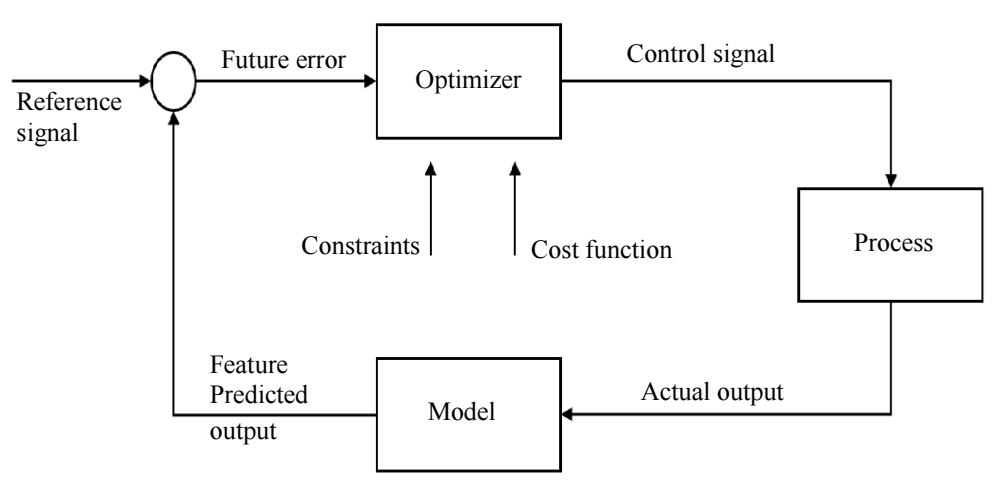

Fig. 1: Model predictive control schematic

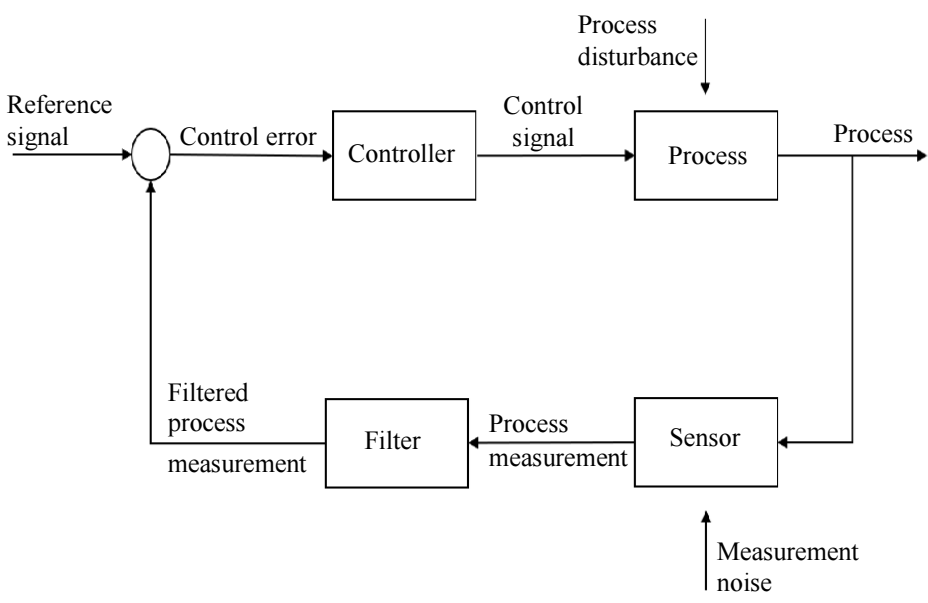

Fig. 2: Proportional integral derivative schematic

\section{Vehicle Modelling}

In this thesis several dynamic models were presented which are used for control design and simulation. Set of models are presented which are suitable for modelbased control design. Over the past decades studies has been carried out extensively on vehicle dynamics (Rajamani, 2012).

From the above sketch of the vehicle body coordinate system, the force acting on car's center of gravity with the body fixed frame of reference. $x, y$ and $z$ are longitudinal, lateral and vertical axes respectively. The longitudinal and lateral forces acting on center of gravity of the vehicle are $F_{x}$ and $F_{y}$ and the rotating moment about $z$ axis is $M_{z}$ :

$$
\begin{aligned}
& m \ddot{x}=F_{x}+m \ddot{y} \dot{\varphi}, \\
& m \ddot{x}=F_{y}-m \dot{y} \dot{\varphi}, \\
& I_{z} \ddot{\varphi}=M_{z},
\end{aligned}
$$

where, $I$ is the vehicle's moment of inertia about $z$ axis and $m$ is the vehicle mass:

$$
\begin{aligned}
& \dot{X}=\dot{x} \cos \varphi-\dot{y} \sin \varphi \\
& \dot{Y}=\dot{x} \sin \varphi-\dot{y} \cos \varphi
\end{aligned}
$$

\section{Model Predictive Control Problem}

A Model Predictive Controller has been designed for the steering control. Such that the vehicle stays within the lanes follows the desired path as close as possible. The model is discretize following the Euler method to obtain a finite dimensional optimal control problem: (Alberto Bemporad, 2002)

$$
\begin{aligned}
& \xi(k+1)=f_{\mu}^{d t}(\xi(k), u(k)) \\
& u(k)=(k-1)+\Delta u(k) \\
& \eta(k)=h(\xi(k))
\end{aligned}
$$


Where:

$$
\begin{aligned}
& u(k)=\left[\delta_{f}, T_{b_{f, l}}, T_{b_{f, l}}, T_{b_{r, l}}, T_{b_{r, r}},\right] \\
& \Delta u(k)=\left[\Delta \delta_{f}(k), \Delta T_{b_{f, l}}, \Delta T_{b_{f, l}}, \Delta T_{b_{r, l}}, \Delta T_{b_{r, r}}\right]
\end{aligned}
$$

considering the cost function:

$$
\begin{aligned}
& \mathrm{J}\left(\xi(t), \Delta U_{t}\right)=\sum_{i=1}^{H_{p}}\left\|\eta_{t+i, t}-\eta_{\text {ref }_{t+i, t}}\right\|_{Q}^{2} \\
& +\sum_{i=0}^{H_{c}-1}\left\|\Delta u_{t+i, t}\right\|_{R}^{2}+\sum_{i=0}^{H_{c}-1}\left\|u_{t+i, t}\right\|_{S}^{2}
\end{aligned}
$$

$\Delta U_{t}$ is the optimization vector at time $t, H_{p}$ and $H_{c}$ denote the output prediction horizon and control horizon.

In the standard MPC designs, we consider $H_{p}>H_{c}$ the control signal is assumed to be constant for all $H_{c} \leq \mathrm{t} \leq H_{p}$, reference signal $\eta_{\text {ref }}$ represents the desired output.

Based on the optimization problem we considered the linear time invariant model predictive control:

$$
\begin{aligned}
& \min _{\Delta U_{t}} J\left(\xi_{t}, U_{t}\right) \\
& \xi_{t+1, t}=A_{t} \xi_{k, t}+B_{t} u_{k, t}+d_{k, t} \\
& k=t, \ldots ., t+H_{p}-1
\end{aligned}
$$

At the current state, the equations are a discrete linear approximation, based on new state and input measurements the linear model is computed:

$$
\begin{aligned}
& {\left[\begin{array}{l}
\alpha_{f_{k, t}} \\
\alpha_{r_{k, t}}
\end{array}\right]=C_{t} \xi_{k, t}+D_{t} u_{k, t}+e_{k, t}} \\
& \eta_{k, t}=h\left(\xi_{k, t}\right) \\
& k=t+1, \ldots ., t+H_{p} \\
& u_{k, t}=u_{k-1, t}+\Delta u_{k, t} \\
& k=t+1, \ldots ., t+H_{p}-1
\end{aligned}
$$

Linear time invariant solves the problem at each time step, once the solution for a problem is obtained, we can compute the input command as (Borrelli, 2003):

$$
u(k)=u(k-1)+\Delta u_{t, t}
$$

where, $\Delta u_{t, t}$ is the vector of the first five given elements of the $\Delta U_{i}$ :

$$
\begin{aligned}
& u_{t-1, t}=u(t-1) \\
& u_{f, \min } \leq u_{k, t} \leq u_{f, \max } \\
& k=t, \ldots ., t+H_{p}-1 \\
& \Delta u_{f, \min } \leq \Delta u_{k, t} \leq \Delta u_{f, \max } \\
& k=t, \ldots ., t+H_{c}-1
\end{aligned}
$$

Through the constraints the stability of the closed loop system is enforced and the function of the time required to setup the problem: (Francesco Borrelli, 2006)

$$
\begin{aligned}
& \alpha_{f_{\min }} \leq \alpha_{f_{k, t}} \leq \alpha_{f_{\max }} \\
& \alpha_{r_{\min }} \leq \alpha_{r_{k, t}} \leq \alpha_{r_{\max }} \\
& k=t, \ldots ., t+H_{p}-1
\end{aligned}
$$

The performance of the linear MPC is not accepted without the above constraints and sometimes it may be unstable. This is because the model we are using is a simple linear model and may not be able to predict the slope change in tire characteristics. We can overcome this problem by adding constraints to the optimization problem. Tire slip ratio and slip angle are required to maintain the vehicle stability (Bakker, 1987).

\section{Steering Control Using MPC}

Steering control for the vehicle is done using the Lane Keeping Assist (LKA) system (Valerio Turri, 2013). The lateral movement of the vehicle in the lanes is controlled using the non-linear time invariant system. Lane Keeping Assist is a control system that makes sure the vehicle is travelling within the designated lanes of the road. If the vehicle is deviated from the center of the lane, Lane Keeping Assist system detects and adjusts the steering angle to restore the position of the vehicle inside the lane (Steven and Keen, 2006).

\section{Simulink Model of the Ego Car}

Using the linear time invariant the lateral vehicle dynamics are represented. The input, output and state variables are stated with the line time invariant, state variables are assumed to be zero in the initial conditions. The state variables are same as the input variables, longitudinal velocity set to $6.70 \mathrm{~m} / \mathrm{s}$ :

- Input variables: $\quad V_{y}$ (Lateral velocity) 


\section{- Output variables: $\quad r$ (yaw angle rate) \\ - State variables: \\ $\delta$ (steering angle) \\ $V_{y}$ (Lateral velocity) \\ $r$ (yaw angle rate)}

The State space model, $G(s)$, of the lateral vehicle dynamics is:

$$
\begin{aligned}
& A=\left[\begin{array}{cc}
-\frac{(2 C f+2 C r) V x}{m} & \frac{-V x-(2 C f * l f-2 C r * l r) V x}{m} \\
\frac{-(2 C f * l f-2 C r * l r) V x}{I z} & \frac{-\left(2 C f *(l f)^{2}-2 C r *(l r)^{2}\right) V x}{I z}
\end{array}\right] \\
& B=\left[\begin{array}{ll}
\frac{2 C f}{m} & \frac{2 C f * l f}{I z}
\end{array}\right]
\end{aligned}
$$

\section{Lateral Vehicle Dynamics}

The lateral vehicle dynamics of the ego car using the bicycle model. Yaw moment inertia of the vehicle is calculated and the longitudinal distance from the center of gravity to the front and rear tires is measured. Cornering stiffness of the front and rear tires is calculated (DaiB, 1994).

\section{Cornering Stiffness Calculation}

When the cornering forces acting on the tires at a turn, the ability of the tire to resist the deformation in the shape is the cornering stiffness. The stiffness of the tire depends upon the flexibility, more flexible the tire is less stiff. When the vehicle is moving at high speeds at the corners, tire develops a lateral force and will also experience a lateral slip (Dixon, 1996).

Slip angle $\alpha$, is the difference between the direction of travel and direction of heading. When the camber angle is zero, the lateral force $F_{y}$ the cornering force. When the slip angle is less than $8^{\circ}$, then the relationship is linear, so that the cornering can be defined as:

$F_{y}=C_{\alpha} \cdot \alpha$ where, $C_{\alpha}$ is the tire cornering stiffness and it depends on the tire size, type, cord angles of the tire.

\section{Center of Gravity Calculation}

The Center of Gravity (CG) height can be calculated by lifting the rear axle of the car 10 inches and weighing the front axle of the car. Using the rules of trigonometry, the CG height is found by considering the law of tangents and Pythagorean theorem.

In the Fig. 3, it shows when rear axle of the vehicle is lifted to 10 inches and the wheelbase is now at an angle with respect to the ground:

$h_{C G}=R_{F}\left(\frac{a}{l}\right)+R_{R}\left(\frac{b}{l}\right)+\left(\frac{W_{F} l-W_{\alpha}}{W \tan \theta}\right)$

In the Fig. 4, the center of gravity height from the ground for Chevy Bolt 2017 has been calculated using different parameters. All vehicle components must be considered to get a precise location of the CG including the non-suspended mass, engine, payload, gearbox and chassis. We need to calculate and set the CG coordinate and the associate mass for each component considered.

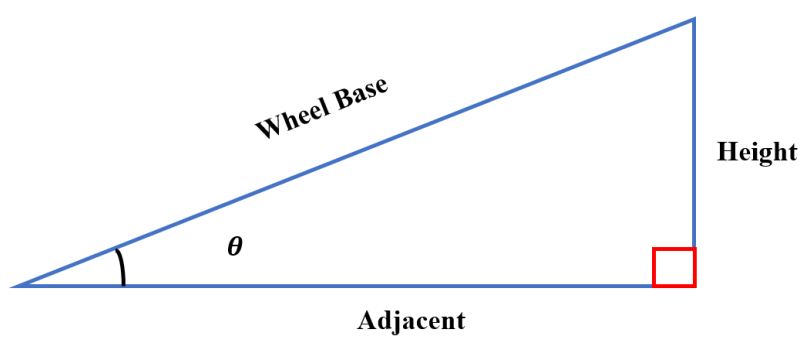

Fig. 3: Wheel base when car lifted 10 inches

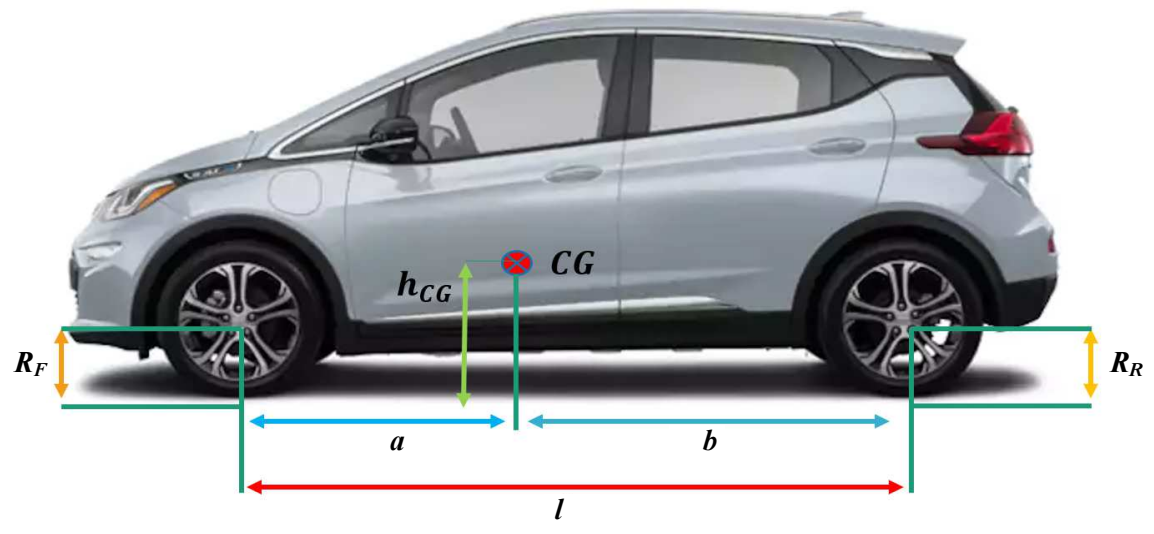

Fig. 4: CG calculation for Chevy bolt 2017 


\section{Yaw Moment of Inertia Calculation}

Inertia caused by each component is calculated:

$$
\begin{aligned}
& I x x_{i}=m_{i}\left(Y_{i}^{2}+Z_{i}^{2}\right) \\
& I y y_{i}=m_{i}\left(X_{i}^{2}+Z_{i}^{2}\right) \\
& I z z_{i}=m_{i}\left(X_{i}^{2}+Y_{i}^{2}\right)
\end{aligned}
$$

The inertia $I x, I y$ and $I z$ is the sum of each component's inertia:

$$
\begin{aligned}
& I x=\sum_{k=1}^{n}\left(Y_{i}^{2}+Z_{i}^{2}\right) \\
& I y=\sum_{k=1}^{n}\left(X_{i}^{2}+Z_{i}^{2}\right) \\
& I z=\sum_{k=1}^{n}\left(X_{i}^{2}+Y_{i}^{2}\right)
\end{aligned}
$$

Only the suspended mass needs to consider for the inertia around the $X$ and $Y$ axis, but we need to consider the suspended and non-suspended mass for the inertia around $Z$ axis.

\section{Sensor Dynamics and Curvature Preview}

Dynamics for lateral deviation: Dynamics for relative yaw angle: Curvature preview look ahead time:

$$
\begin{aligned}
& \dot{e}_{2}=V_{x \rho} \\
& \dot{e}_{1}=V_{x} e_{2}+V_{y} \\
& T=1 \text { second } \\
& T s=1 \\
& 10 \text { steps }
\end{aligned}
$$$$
\text { Prediction Horizon: }
$$

\section{Adaptive Cruise Control Using MPC}

Relative speed and relative distance are maintained by the ego car with the Adaptive Cruise Control system. With the longitudinal acceleration and deceleration control capabilities it reduces the driver's longitudinal control responsibility and tries to maintain relative speed and relative distance. Initially the driver sets the speed for the ego car to follow (Re, 2013). The ego car is equipped with a sensor, such as radar which recognizes the vehicles and objects in front of it. In the ACC system the radar measures the distance between the lead car and the ego car, if the distance between them is less than safe distance it reduces the speed until it reaches the safe distance. The ego car follows the driver set speed only when the distance between the lead car and ego car is above the safe distance (Schutter, 2008):

- If $D_{\text {rel }} \geq D_{\text {safe, }}$, then speed control mode is active. Goal is to maintain driver set velocity, $V_{\text {set }}$
- If $D_{\text {rel }}<D_{\text {safe, }}$, then spacing control mode is active. Goal is to maintain safe distance, $D_{\text {safe }}$

The car is equipped with an Adaptive Cruise Control system has a sensor, such as radar. In real-time radar measurements ACC system decides which mode to be used. The goal is to follow the driver set speed if the distance between the lead car and ego car is above the safe distance.

\section{Simulink Model of the Ego Car}

Acceleration of the lead varies according to the sine wave, acceleration of the ego car is set to zero initially. ACC system outputs an acceleration control signal for the ego car. For the lead vehicle and ego vehicle the dynamics between the acceleration and velocity is modelled as:

$$
G=\frac{1}{s(0.5 s+1)}
$$

\section{Vehicle Dynamics}

Longitudinal dynamics model with a nonlinear single-track augmented model is used to represent the vehicles lateral and longitudinal dynamics. The longitudinal and lateral tire forces are calculated. The steering angle projection is characterized by the nonlinear single-track augmented model (Shengbo Li, 2011):

$$
\begin{aligned}
{\left[\begin{array}{c}
\sum F_{X} \\
\sum F_{Y} \\
\sum M_{Z}
\end{array}\right] } & =\left[\begin{array}{cc}
-\sin \delta_{f} & -\sin \delta_{r} \\
\cos \delta_{f} & \cos \delta_{r} \\
l_{f} \cos \delta_{f} & -l_{r} \cos \delta_{r}
\end{array}\right]\left[\begin{array}{l}
F_{Y F} \\
F_{Y R}
\end{array}\right] \\
& +\left[\begin{array}{cc}
\cos \delta_{f} & \cos \delta_{r} \\
\sin \delta_{f} & \sin \delta_{r} \\
-l_{f} \sin \delta_{f} & -l_{r} \sin \delta_{r}
\end{array}\right]\left[\begin{array}{l}
F_{X F} \\
F_{X R}
\end{array}\right]
\end{aligned}
$$

Newton-Euler dynamics equations of motions:

$$
\left[\begin{array}{c}
m v(\beta+r) \\
\dot{v} v \\
\dot{J} r
\end{array}\right]=\left[\begin{array}{ccc}
\sin \beta & \cos \beta & 0 \\
\cos \beta & \sin \beta & 0 \\
0 & 0 & 1
\end{array}\right]\left[\begin{array}{c}
F_{X} \\
F_{Y} \\
M_{Z}
\end{array}\right]
$$

Equations of kinematics/geometry:

$$
\begin{aligned}
& \tan \beta_{f}=\tan \beta+\frac{l_{f} r}{v \cos \beta} \\
& \tan \beta_{r}=\tan \beta+\frac{l_{r} r}{v \cos \beta}
\end{aligned}
$$

In vector form tire center velocities are presented:

$\overrightarrow{v_{r}}=v \cos \beta \vec{i}+\left(v \sin \beta-r l_{r}\right) \vec{j}$

$\overrightarrow{v_{f}}=v \cos \beta \vec{i}+\left(v \sin \beta-r l_{f}\right) \vec{j}$ 


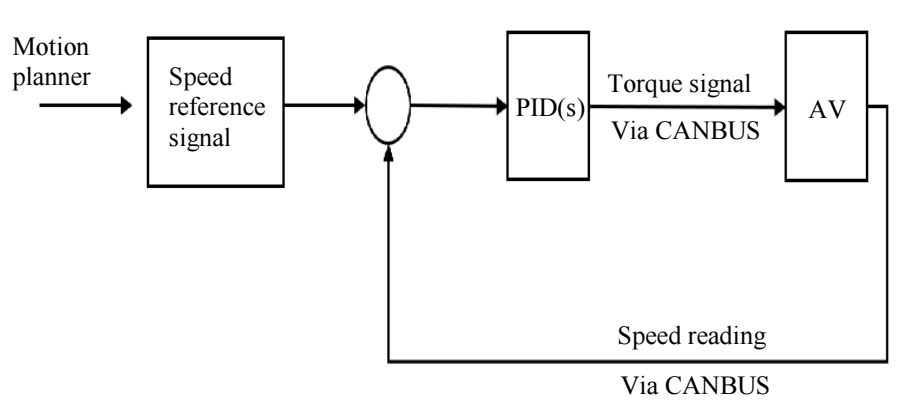

Fig. 5: Adaptive cruise control system

\section{Linear Model for MPC Structure}

The linear model represented the inter-vehicle dynamics in the MPC structure (Dominik Moser, 2015):

$$
\begin{aligned}
& x(k+1)=A x(k)+b u(k)+E w(k) \\
& y(k+1)=c x(k)+F
\end{aligned}
$$

Acceleration of the ego car, relative speed and distance error are the outputs of the model. Initial position of the lead car and the ego car is set, lead car varies the acceleration as modelled, ego car initially accelerates fast to reach the driver set speed when it approaches the lead car it reduces the speed. The ego car tries to maintain the driver set speed if the spacing control mode is not active. The distance between the lead car and ego car is varied, we can observe the spacing error in the simulation results. The distance between the lead car and ego car is set based on the spacing control distance, which is decided based upon the simulation results.

\section{Sensor Dynamics and Curvature Preview}

Dynamics for lateral deviation: Dynamics for relative yaw angle: Curvature Look ahead time:

Sample time:

Prediction Horizon:

$$
\begin{aligned}
& \dot{e}_{2}=V_{x \rho} \\
& \dot{e}_{1}=V_{x} e_{2}+V_{y} \\
& T=1 \mathrm{sec} \\
& T s=1 \\
& 10 \text { steps }
\end{aligned}
$$

In the Fig. 4, the controller schematic for adaptive cruise control system, the CANBUS cables are connected to vehicle for speed reading.

\section{Results}

\section{Lane Keeping Assist}

The state space model of the later vehicle dynamics:

$$
\begin{aligned}
A & =\left[\begin{array}{cc}
-\frac{(2 C f+2 C r) V x}{m} & \frac{-V x-(2 C f * l f-2 C r * l r) V x}{m} \\
\frac{-(2 C f * l f-2 C r * l r) V x}{I z} & \frac{-\left(2 C f *(l f)^{2}-2 C r *(l r)^{2}\right) V x}{I z}
\end{array}\right] \\
B & =\left[\begin{array}{ll}
\frac{2 C f}{m} & \frac{2 C f * l f}{I z}
\end{array}\right]
\end{aligned}
$$

Center of gravity has been calculated and located for the Chevy Bolt. Longitudinal distance from the center of gravity to the front axle and rear axle are measured. Calculating the center of gravity for each component and measuring the distance from vehicle center of gravity to each individual component center of gravity gives the yaw moment inertia of the vehicle. Cornering stiffness of the front and rear tires is calculated and mass of the vehicle is known from the vehicle user's manual:

- $m=1625 \mathrm{~kg}$

- $\quad I z=2650 \mathrm{mNs}^{2}$

- $l f=1.214 \mathrm{~m}$

- $l r=1.386 \mathrm{~m}$

- $C f=18450 \mathrm{~N} / \mathrm{rad}$

- $C r=32400 \mathrm{~N} / \mathrm{rad}$

In the Fig. 5, the vehicle trajectory follows the reference trajectory closely, when the vehicle travels along a straight line, then $V_{y}=0$, both $Y_{r e f}$ and $\varphi_{\text {ref }}$ are perfectly tracked. The relative yaw angle and lateral deviation both converge to zero, the ego car follows the road closely due to the relations $\dot{e}_{2}=V_{x \rho}$ and $\dot{e}_{1}=V_{x} e_{2}+$ $V_{y}$ based on the previewed curvature (Dekkata, 2018).

\section{Adaptive Cruise Control}

Lead vehicle and ego vehicle dynamics between acceleration and velocity are modelled as:

$$
G=\frac{1}{s(0.5 s+1)}
$$

In Fig. 7, Initial position and velocity of the ego vehicle and lead vehicle:

- $x_{o \_}$lead $=50 \mathrm{~m}$; initial position for lead car

- $v_{o}$ lead $=25 \mathrm{~m} / \mathrm{s}$; initial velocity of the lead car

- $x_{o_{-}}$ego $=10 \mathrm{~m}$; initial position of the ego car

- $x_{o \_}$ego $=20 \mathrm{~m} / \mathrm{s}$; initial position of the lead car

The safe distance between the lead car and the ego car is a function of the ego car velocity, $V_{\text {ego }}$ : 


$$
D_{\text {safe }}=D_{\text {default }}+T_{\text {gap }} * V_{\text {ego }}
$$

$D_{\text {default }}=10$; is the standstill spacing in $m$ $T_{\text {gap }}=1.4$; time gap between the vehicles,

Where:

$$
V_{\text {set }}=30 ; \quad \text { driver set velocity } \mathrm{m} / \mathrm{s}
$$

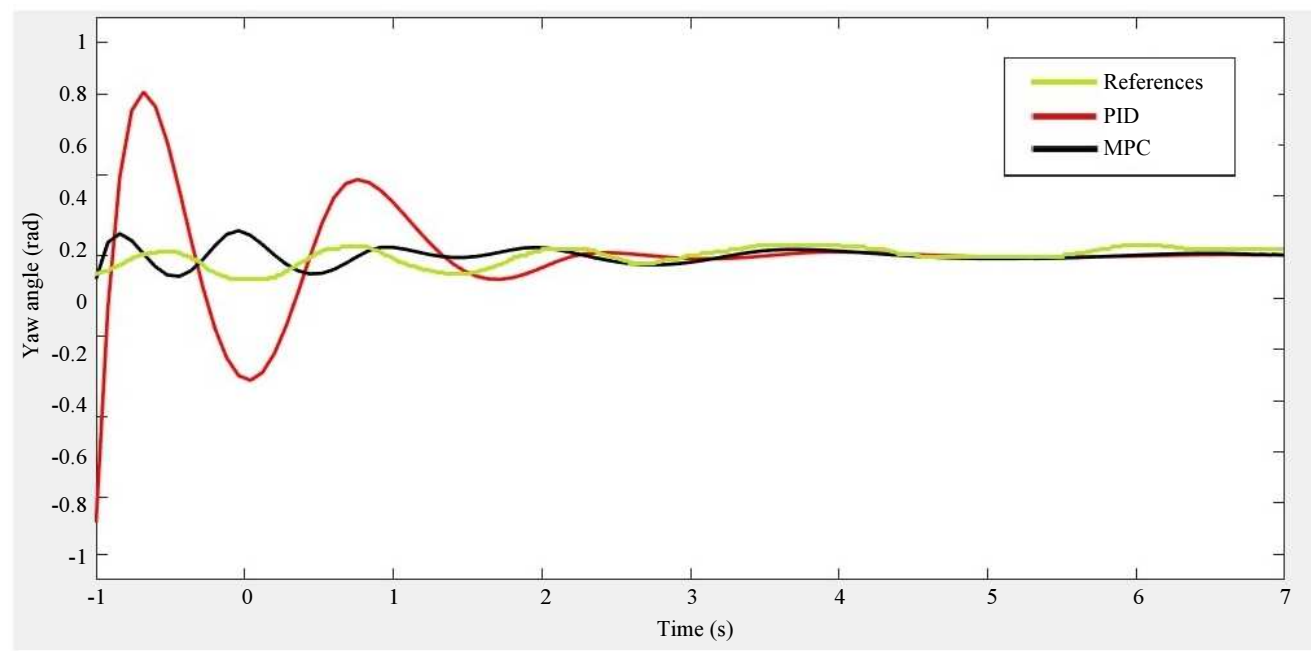

Fig. 6: MPC closely following the reference
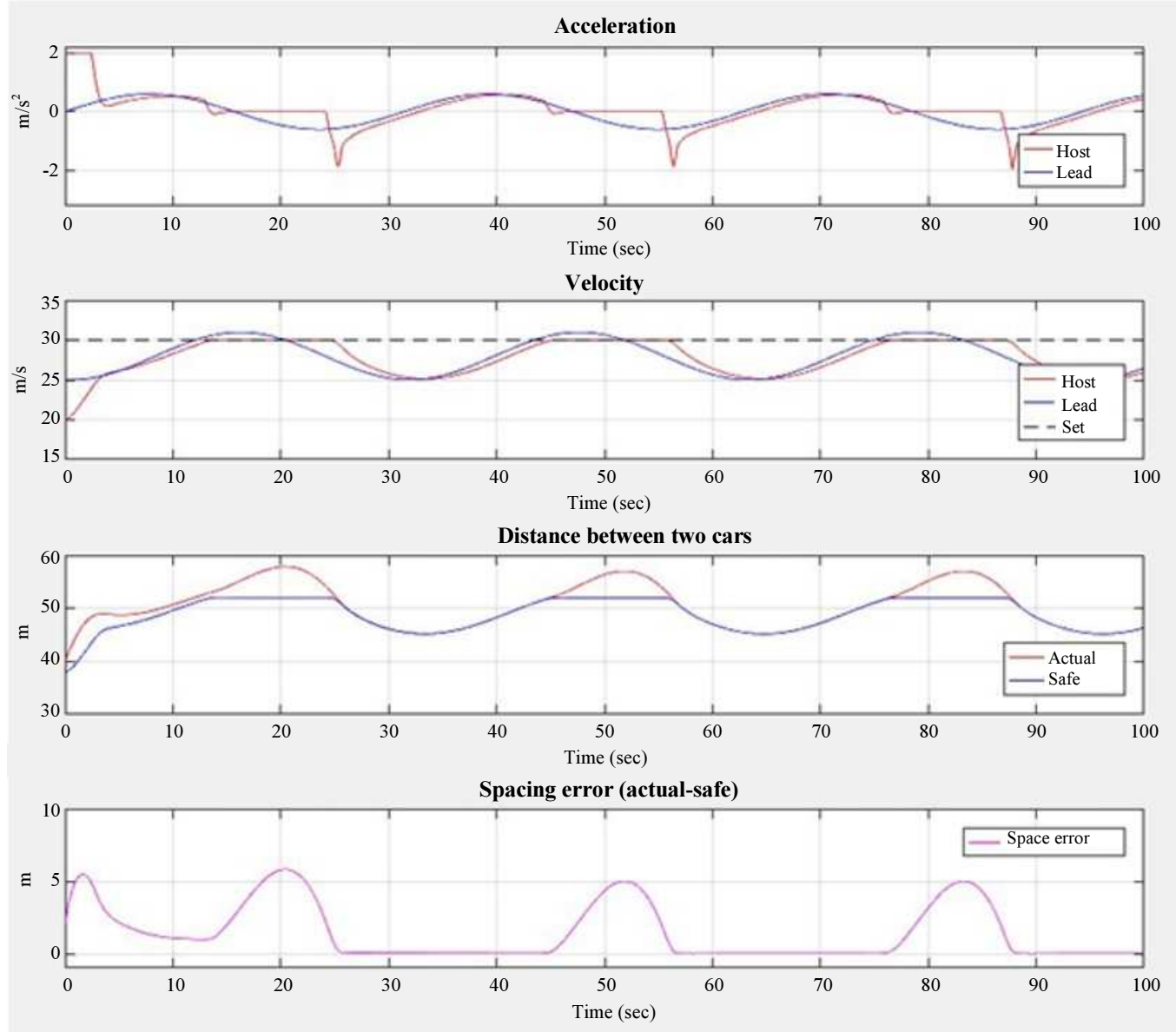

Fig. 7: Spacing distance is set to $5 \mathrm{~m}$ 

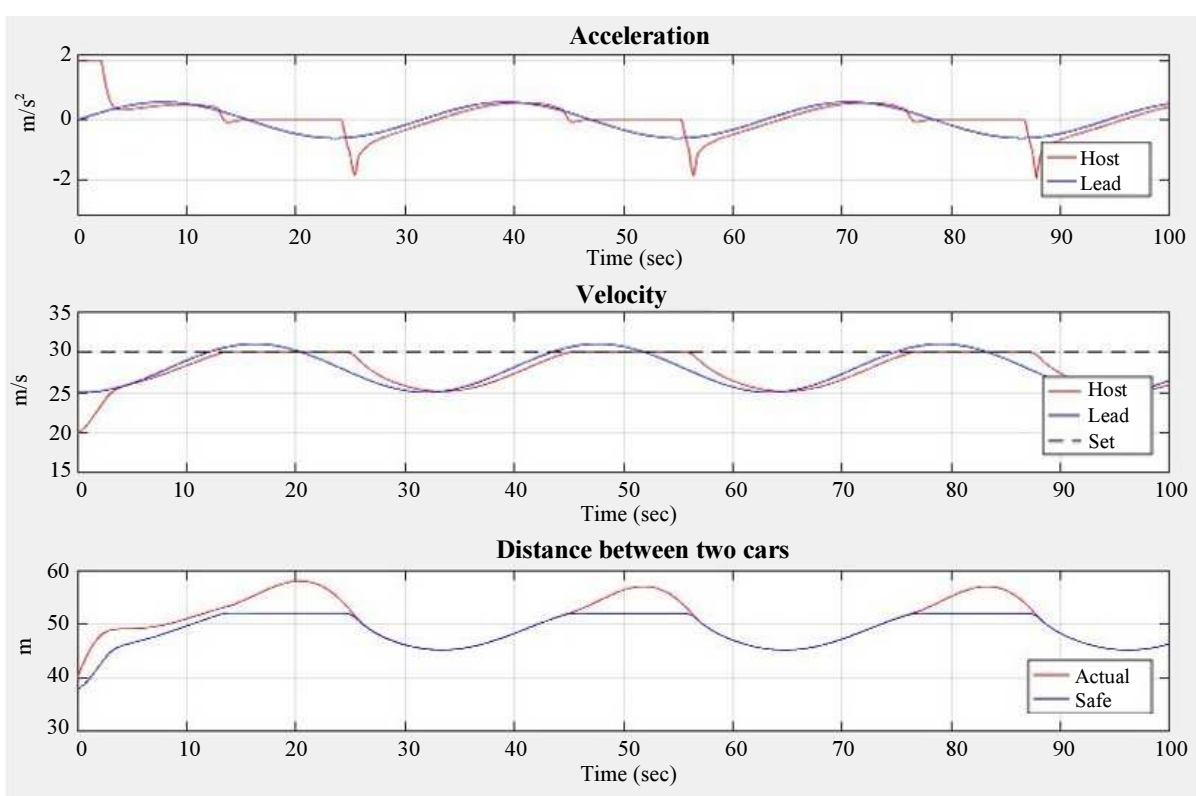

Spacing error (actual-safe)

$\Xi$

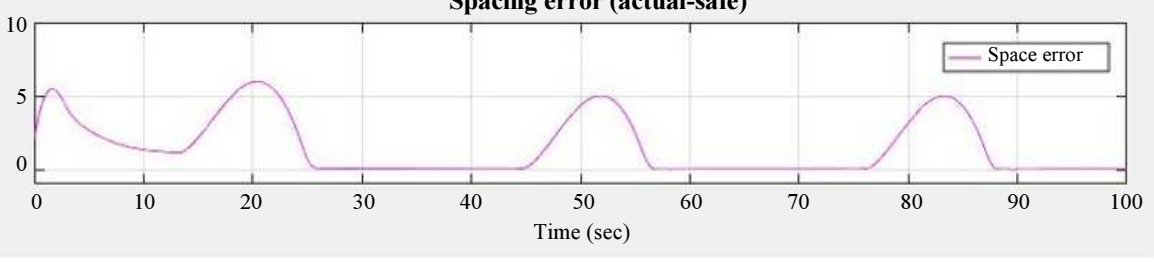

Fig. 8: Spacing distance is set to $10 \mathrm{~m}$

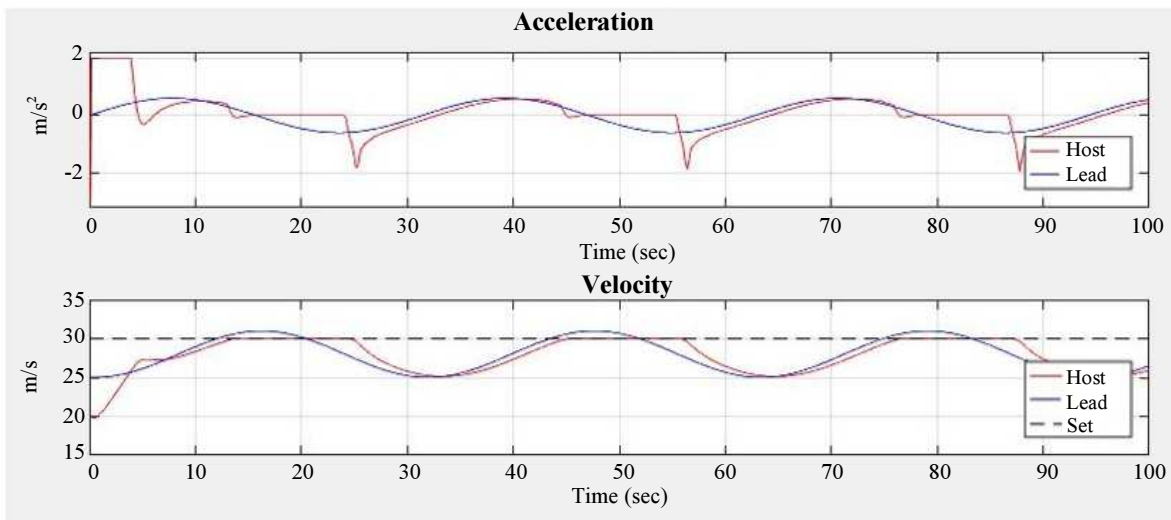

Distance between two cars

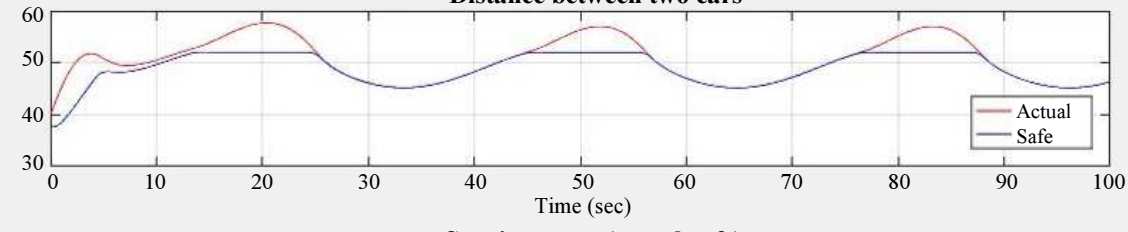

Spacing error (actual-safe)

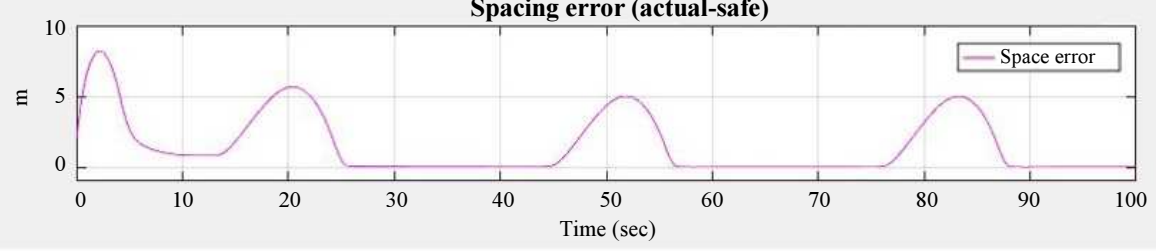

Fig. 9: Spacing distance is set to $15 \mathrm{~m}$ 

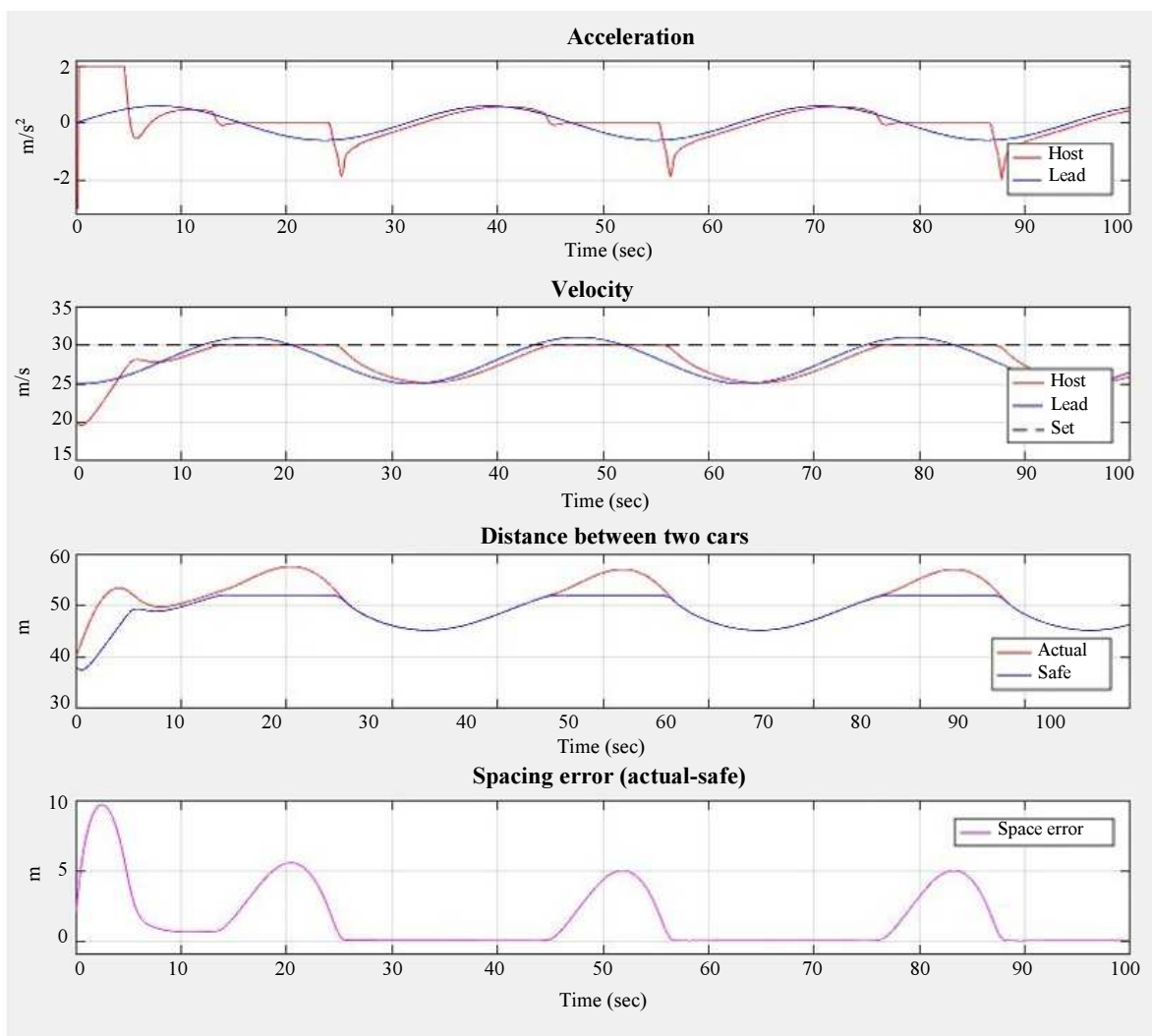

Fig. 10: Spacing distance is set to $20 \mathrm{~m}$

Vehicle dynamics physical limitations were considered, acceleration is constrained to range:

- Minimum acceleration of ego car $=-3 \mathrm{~m} / \mathrm{s}^{2}$

- Maximum acceleration of ego car $=2 \mathrm{~m} / \mathrm{s}^{2}$

Initially, ego car accelerates fast to reach the driver set velocity, but the lead car accelerates slowly, to maintain the safe distance ego car decelerates and maintains a safe distance. Ego car maintains the driver set velocity when the lead car is at a safe distance.

When the lead car reduces speed, the spacing error increases and the ego car reduces speed. The acceleration and deceleration sequence repeats. Controller makes sure the distance between the two vehicles is always greater than the safe distance, when the distance is larger than the ego car travels at driver set velocity.

Figures 7 and 8, Spacing error is almost negligible when the distance is increased from 5 to $10 \mathrm{~m}$ between the lead car and the ego car. In Fig. 9 and 10, Spacing error increased when the distance increased from 15 to $20 \mathrm{~m}$ between the lead car and the ego car.

\section{Conclusion}

PID control has a wide range of applications for the systems having single-input single-output, but MPC is capable of even handling the high models, which have multi-input multi-output. In the Fig. 6, the reference, MPC and PID are compared and future set points are known for MPC, so it is much better than PID, because MPC plans controls by looking ahead. MPC has smoother control signal with less propagation of noise through controller than PID. Linear MPC with a kinematic model is used as the vehicle is moving at relatively low speeds. MPC operates at a high-level hierarchy while the actuation of throttle and brake commands are executed through dedicated low-level controllers. Car follows the reference trajectory almost as closely as possible using PID, but MPC simulation results are comparatively better than the PID's. The velocity gradient for maximum acceleration and deceleration have been tuned for passenger comfort to avoid jerking. There are less disturbances if the Lane Keeping Assist and Adaptive Cruise Control systems are used separately, but successfully implemented both Lane Keeping Assist and Adaptive Cruise Control systems together by adjusting the controller gains.

\section{Acknowledgement}

The authors are thankful to Center for Advance Transportation Mobility for providing funds to pursue this research. We are also grateful to the North Carolina A\&T State University for providing the Infrastructure. 


\section{Author's Contributions}

Sai Charan Dekkata: Designed the PID controller and developed the MPC to compare the simulation results.

Sun Yi: Developed the theoretical framework and supervised the research.

\section{Ethics}

This article is an original research paper. There are no ethical issues that may arise after the publication of this manuscript.

\section{References}

Alberto Bemporad, M.M., 2002. The explicit linear quadraticregulator for constrained systems. Automatica, PERGAMON.

Bakker, E.A., 1987. Tire modelling for use in vehicle dynamics studies. SAE MOBILUS.

Borrelli, F., 2003. Constrained optimal control of linear and hybrid systems. Springer.

DaiB, U.K., 1994. Observation of lateral vehicle dynamics. Pergamon, Elsevier Science Publication.

Dave Misir, H.A., 1994. Design and analysis of a fuzzy proportional-integral-derivative controller. Fuzzy Sets Systems.

Dekkata, S.C., 2018. Steering and adaptive cruise control for autonomous vehicles using model predictive control. Greensboro, NC - USA: Proquest.

Dixon, J.C., 1996. Tires, suspension and handling. SAE International.

Dominik Moser, H.W., 2015. Cooperative adaptive cruise control applying stochastic linear model predictive control strategies. European Control Conference. Linz, Austria: ECC.
Francesco Borrelli, A.B., 2006. An MPC/Hybrid System approach to traction control. IEEE Trans. Control Syst. Technol.

Rajamani, R., 2012. Vehicle dynamics and control. Springer.

Re, T.S., 2013. A model predictive cooperative adaptive cruise control approach. American Control Conference. Washington, DC: ACC.

Schutter, D.C., 2008. Adaptive cruise control for a SMART car: A comparision benchmark for MPC_PWA control methods. IEEE Trans. Control Syst. Technol.

Shengbo Li, K.L., 2011. Model predictive multi-object vehicular adaptive cruise control. IEEE Trans. Control Syst. Technol.

Steven, D. and D.J. Keen, 2006. Steering control using model predictive control and multiple internal models. Proceedings of the International Symposium on Advanced Vehicle Control. Taipei, Taiwan: AVEC .

Valerio Turri, A.C., 2013. Linear model predictive control for lane keeping and obstacle avoidance on low curvature roads. Proceedings of the International IEEE Annual Conference on Intelligent Transportation Systems. The Hague, Netherlands: ITSC.

Yiqi Gao, A.G., 2014. Robust nonlinear predictive control for semiautonomous ground vehicles. Proceedings of the American Control Conference. Portland, Oregon. 